\title{
Dynamics and stability of a class of low Reynolds number swimmers near a wall
}

\author{
Yizhar Or* and Richard M. Murray \\ Department of Control and Dynamical Systems, California Institute of Technology, \\ 1200 E California Boulevard, Pasadena, California 91125, USA
}

(Received 18 November 2008; revised manuscript received 10 February 2009; published 13 April 2009)

\begin{abstract}
We study the dynamic stability of low Reynolds number swimming near a plane wall from a controltheoretic viewpoint. We consider a special class of swimmers having a constant shape, focus on steady motion parallel to the wall, and derive conditions under which it is passively stable without sensing or feedback. We study the geometric structure of the swimming equation and highlight the relation between stability and reversing symmetry of the dynamical system. Finally, our numerical simulations reveal the existence of stable periodic motion. The results have implications for design of miniature robotic swimmers, as well as for explaining the attraction of micro-organisms to surfaces.
\end{abstract}

DOI: 10.1103/PhysRevE.79.045302

\section{INTRODUCTION}

The motion of micro-organisms [1], as well as of tiny robotic swimmers [2], is governed by low Reynolds number $(\mathrm{Re})$ hydrodynamics, where viscous effects dominate and inertial effects are negligible [3-5]. Many works have analyzed the dynamics of low-Re swimming in unbounded fluid by exploiting its special geometric structure, namely, the gauge symmetry, which implies that the equation of motion is invariant under rigid body transformations [6]. However, in reality, swimmers often move in a confined environment, where their hydrodynamic interaction with the boundary is complicated to model $[7,8]$. The loss of gauge symmetry then raises problems of control-theoretic nature, such as steering to a desired direction and dynamic stability of the motion under perturbations.

In this Rapid Communication, we investigate the dynamic stability of low-Re swimming near a plane wall from a control-theoretic viewpoint. Unlike classical models such as [9] that utilize shape changes to induce self-propulsion, we focus on a special class of swimmers, having an apparently constant shape. Examples of theoretical models of such swimmers are the twirling torus [10] and the surface treadmiller [11], inspired by actin-based cell motility [12]. Our model considers a swimmer which is propelled by rotating spheres and performs planar motion. We formulate the dynamics of swimming near a wall as a control system [13], study its underlying geometric structure, and show that it is associated with a reversing symmetry [14]. We analyze swimming motion parallel to the wall and prove that the reversing symmetry implies marginal stability of this motion under perturbations, leading to periodic solutions of wavelike and spiral motions. Next, we show that by breaking the reversing symmetry, one can obtain passive asymptotic stability. Finally, we show the existence of stable periodic motion along the wall, associated with a Hopf bifurcation [15].

The implications of our results, and, in particular, the passive stabilization of swimming parallel to wall, are twofold. In the context of artificial swimmers for biomedical applications [2], our analysis shows that they can be steered in con-

\footnotetext{
*izi@cds.caltech.edu
}

PACS number(s): 47.63.mf, 45.80.+r, 47.10.Fg, 11.30.Er

fined environments by using open-loop commands only, without requiring on-board sensing and control for stabilization. In the context of biological systems, the results can contribute to ongoing study on the accumulation of swimming micro-organisms near solid surfaces [1]. This phenomenon was observed experimentally in bacteria [16] and sperm cells [17] and was verified via numerical simulations in $[8,18]$ confirming that it is caused primarily by hydrodynamic effects rather than by other factors such as chemotaxis [1]. The pioneering work in [19] presents a simple physical explanation for the attraction of the swimmer and its alignment parallel to the wall by modeling the swimmer as a point acted by a force dipole. However, this model does not capture the convergence to a nonzero distance from the wall and does not analyze dynamic stability of the motion. Our work proposes an extension of this model, which is discussed further in the sequel.

\section{KINEMATIC MODEL}

We consider a simple model of a microswimmer comprised of $n$ rigid spheres of radius $a$ connected by a rigid structure of thin rods called the body of the swimmer. The swimmer is submerged in a quiescent viscous fluid which is bounded by an infinite plane wall at $y=0$ [Fig. 1]. The spheres' centers lie within the $x y$ plane, and all motions are assumed to be constrained to that plane. The spheres labeled $1 \ldots m$ are actuated by rotation about their $z$ axis which is fixed to the body, while the rest of the spheres are rigidly

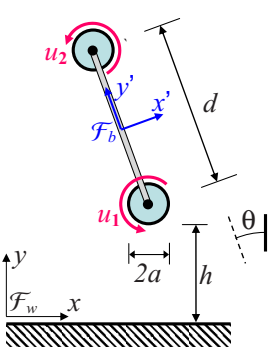

(a)

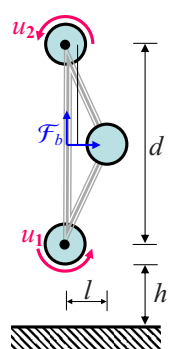

(b)

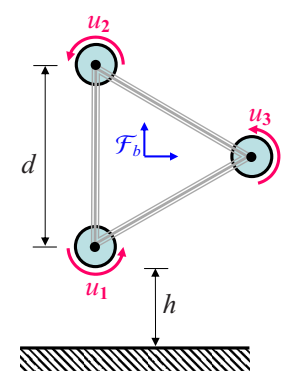

(c)
FIG. 1. (Color online) Drawing of the swimmers. (a) Twosphere swimmer. (b) 2+1-sphere swimmer. (c) Three-sphere swimmer. 
attached to the body. Let $\mathcal{F}_{w}$ be a world-fixed reference frame, and let $\mathcal{F}_{b}$ be a reference frame attached to the swimmer's body. Let $u_{i} \in \mathbb{R}$ be the angular velocity of the $i$ th sphere with respect to the body and denote $\mathbf{u}=\left(u_{1} \ldots u_{m}\right)^{T}$, which is regarded as the swimmer's input. Let $\mathbf{q}=(x, y, \theta)^{T}$ $\in S E(2)$ denote the position and orientation of the body expressed in the world frame $\mathcal{F}_{w}$. Let $\mathbf{r}_{i}$ be the constant position vector of the $i$ th sphere expressed in the body frame $\mathcal{F}_{b}$. Let $\mathbf{V}_{i} \in \mathbb{R}^{2}$ denote the linear velocity of the $i$ th sphere in $x y$ plane, and let $\omega_{i} \in \mathbb{R}$ be its angular velocity about the $z$ axis. The linear velocity of the $i$ th sphere is then given by

$$
\mathbf{V}_{i}=\left(\begin{array}{c}
\dot{x} \\
\dot{y}
\end{array}\right)+\mathbf{D}(\theta) \mathbf{r}_{i} \dot{\theta}, \quad \text { where } \mathbf{D}(\theta)=\left(\begin{array}{cc}
-\sin \theta & -\cos \theta \\
\cos \theta & -\sin \theta
\end{array}\right)
$$

The angular velocity of the $i$ th sphere is given by $\omega_{i}=\dot{\theta}+u_{i}$ for $i=1 \ldots m$ and $\omega_{i}=\dot{\theta}$ for $i=m+1 \ldots n$. Defining $\mathbf{U}=\left(\mathbf{V}_{1}, \ldots \mathbf{V}_{n}, \omega_{1}, \ldots, \omega_{n}\right)^{T}$, the velocity relations above can be written in matrix form as $\mathbf{U}=\mathbf{T} \dot{\mathbf{q}}+\mathbf{E} \mathbf{u}$.

\section{HYDRODYNAMIC FORCES}

In our model, the fluid motion is governed by the quasisteady Stokes equations [3]. Let $\mathbf{f}_{i}$ and $\tau_{i}$ be the force and torque (about the $z$ axis) exerted by the fluid upon the $i$ th sphere and define $\mathbf{F}=\left(\mathbf{f}_{1}, \ldots \mathbf{f}_{n}, \tau_{1}, \ldots, \tau_{n}\right)^{T}$. Let $\mathbf{F}_{b}=\left(\mathbf{f}_{b}, \tau_{b}\right)^{T}$ denote the net force and torque acting on the body. It is then straightforward to show that $\mathbf{F}_{b}$ satisfies $\mathbf{F}_{b}=\mathbf{T}^{T} \mathbf{F}$ [20]. The linearity of Stokes equations implies a linear relation between hydrodynamic forces and velocities given by $\mathbf{F}=\mathbf{R} \mathbf{U}$, where $\mathbf{R}$ is called the resistance matrix, and depends only on the configuration of the particles [3]. Generally, $\mathbf{R}$ cannot be computed exactly, and instead, it is approximated by using scaling arguments. In this work, we adopt the model by Swan and Brady [21], which computes the mobility matrix defined as $\mathbf{M}=\mathbf{R}^{-1}$, for multiple spherical particles in the presence of a plane wall by accounting for far-field hydrodynamic interactions.

\section{EQUATION OF SWIMMING}

Using all the relations above, the requirement that the swimmer's body is force and torque free at all times, $\mathbf{F}_{b}=0$, gives the swimming equation, which formulates the relation between the input velocities and the body velocity as the control system

$$
\dot{\mathbf{q}}=\mathbf{G}(\mathbf{q}) \mathbf{u}, \quad \text { where } \mathbf{G}(\mathbf{q})=\left(\mathbf{T}^{T} \mathbf{R} \mathbf{T}\right)^{-1} \mathbf{T}^{T} \mathbf{R E} .
$$

\section{TWO-SPHERE SWIMMER}

Consider a simple swimmer made of two actuated spheres connected by a thin rigid rod of length $d$ [Fig. 1(a)]. In the rest of this work, we normalize all lengths by the radius $a$ and set $a=1$. The $3 \times 2$ matrix $\mathbf{G}(\mathbf{q})$ is derived according to Eq. (1), where $\mathbf{R}$ is computed by using the model in [21]. The analytical expressions in the components of $\mathbf{G}(\mathbf{q})$ are too lengthy to be detailed here, and numerical values were computed throughout this work by using MATLAB. Note that in unbounded fluid equal and opposite input velocities $u_{1}=-u_{2}$ result in swimming in a straight line along the $x^{\prime}$
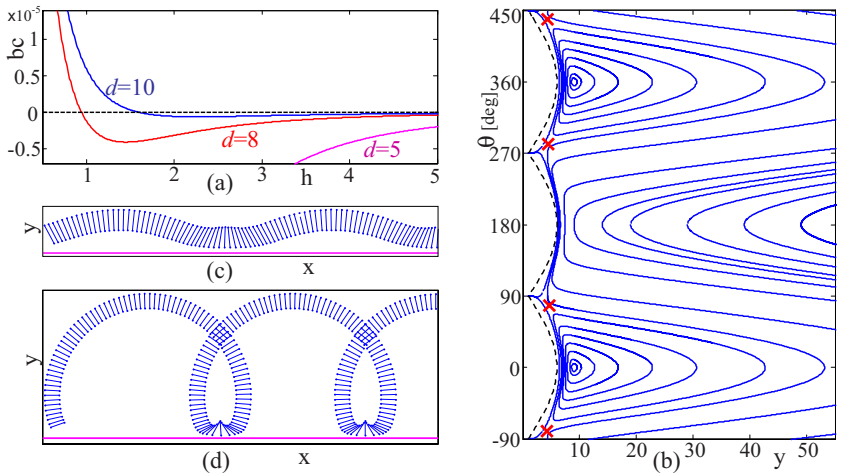

FIG. 2. (Color online) The two-sphere swimmer near wall. (a) Plot of $b c$ as a function of $h$. (b) Phase plot in $(y, \theta)$ plane for $\mathbf{u}_{e}$ $=(1,-1.0068)$. (c) Snapshots of wave-like motion along the wall. (d) Snapshots of spiral-like motion along the wall.

axis due to symmetry of the swimmer about the $x^{\prime} z$ plane [13]. However, the presence of wall breaks this symmetry, and for $u_{1}=-u_{2}$ the swimmer is repelled from the wall and swims along an arc (see Fig. 2 in [13]). Therefore, we consider here the simplest task for this swimmer-steady motion parallel to the wall with fixed orientation and fixed distance from the wall. As a preliminary step, we analyze the geometric structure of the swimming equation. A first observation is that Eq. (1) is invariant under shifting parallel to the wall. Therefore, $\mathbf{G}(\mathbf{q})$ depends only on the coordinates $y$ and $\theta$ and not on $x$. A second observation is that the swimmer possesses a mirror symmetry about the orientation perpendicular to the wall $\theta=0$, which can be formulated as

$$
\mathbf{G}(\mathbf{S q})=-\mathbf{S G}(\mathbf{q}), \quad \text { where } \mathbf{S}=\operatorname{diag}(-1,1,-1) .
$$

The physical meaning of Eq. (2) is that a mirror-reflected swimmer will swim along a reflected trajectory with its input velocities u reversed, which is equivalent to reversing the time. Formally, a relation of form (2) is called a reversing symmetry of a dynamical system [14]. Note that Eq. (2) implies that elements on the second row of $\mathbf{G}(\mathbf{q})$ are odd functions of $\theta$, while elements on the third row of $\mathbf{G}(\mathbf{q})$ are even functions of $\theta$; thus they satisfy

$$
\begin{gathered}
G_{2 j}(\theta=0)=0, \\
\left.\frac{\partial}{\partial y} G_{2 j}\right|_{\theta=0}=0,\left.\quad \frac{\partial}{\partial \theta} G_{3 j}\right|_{\theta=0}=0
\end{gathered}
$$

for $j=1,2$, where $G_{i j}$ is the $(i, j)$ element of $\mathbf{G}(\mathbf{q})$. Constraint (3) implies that for $\theta=0$, the $\dot{y}$ component of $\dot{\mathbf{q}}$ in Eq. (1) vanishes for any $y$ and $\mathbf{u}$. Therefore, for given distance from the wall $y=y_{e}$, taking input velocities $\mathbf{u}_{e}=\alpha\left(-G_{32}, G_{31}\right)$, where $G_{31}, G_{32}$ are evaluated at $(y, \theta)=\left(y_{e}, 0\right)$ and $\alpha \in \mathbb{R}$, the $\dot{\theta}$ component of $\dot{\mathbf{q}}$ also vanishes, and $\dot{\mathbf{q}}$ has the form $\dot{\mathbf{q}}$ $=\left(v_{x}, 0,0\right)$, corresponding to steady motion parallel to the wall with $\theta=0$ [13].

Next, we analyze the dynamic stability of parallel swimming under perturbations [13]. Denoting $\mathbf{q}^{\prime}=(y, \theta)$, the dynamics of $\mathbf{q}^{\prime}$ is given by

$$
\dot{\mathbf{q}}^{\prime}=\mathbf{G}^{\prime}\left(\mathbf{q}^{\prime}\right) \mathbf{u},
$$



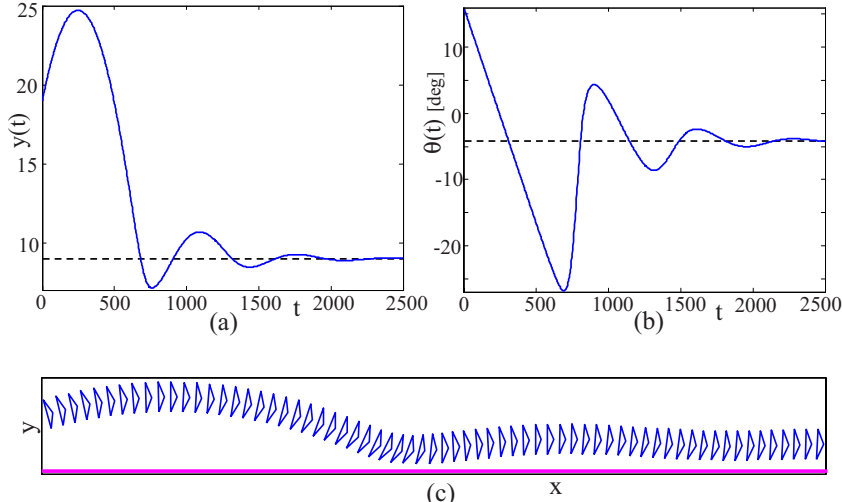

FIG. 3. (Color online) Simulation of the 2+1-sphere swimmer. (a) Plot of $y(t)$. (b) Plot of $\theta(t)$. (c) Snapshots of swimmer's motion.

where $\mathbf{G}^{\prime}$ is the lower $2 \times 2$ block of $\mathbf{G}(\mathbf{q})$ in Eq. (1). By construction, the system [Eq. (5)] has an equilibrium point at $\mathbf{q}_{e}^{\prime}=\left(y_{e}, 0\right)$ under the constant input $\mathbf{u}=\mathbf{u}_{e}$. Formally, $\mathbf{q}_{e}^{\prime}$ is called a relative equilibrium of Eq. (1) [22], as it involves motion along $x$. The local stability of $\mathbf{q}_{e}^{\prime}$ is determined by the linearization of Eq. (5) about $\mathbf{q}_{e}^{\prime}$ given by

$$
\delta \dot{\mathbf{q}}^{\prime}=\mathbf{A} \delta \mathbf{q}^{\prime}, \quad \text { where } \mathbf{A}=\left.\frac{\partial \mathbf{G}^{\prime}\left(\mathbf{q}^{\prime}\right)}{\partial \mathbf{q}^{\prime}}\right|_{\mathbf{q}_{e}^{\prime}} \cdot \mathbf{u}_{e} .
$$

A sufficient condition for asymptotic stability of $\mathbf{q}_{e}^{\prime}$ is that all eigenvalues of $\mathbf{A}$ have negative real part [13]. However, invoking the constraints [Eq. (4)], A takes the form

$$
\mathbf{A}=\left(\begin{array}{ll}
0 & b \\
c & 0
\end{array}\right)
$$

and its characteristic polynomial is $\Delta_{A}(\lambda)=\lambda^{2}-b c$, which precludes the possibility of asymptotic stability (see [13] for intuitive explanation).

The eigenvalues of $\mathbf{A}$ are thus a pair $\pm \lambda$, which can either be imaginary or real, depending on the sign of the product $b c$. In order to distinguish between these two cases, we numerically compute $b c$ (see [13] for details) as a function of the wall-separation $h$, defined as $h=y-a-0.5 d \cos \theta$ [Fig. 1(a)], for different values of $d$. The results, which are plotted in Fig. 2(a), show that when $h$ is sufficiently large, $b c$ is negative, hence $\mathbf{A}$ has a pair of purely imaginary eigenvalues. Moreover, the fact that $\mathbf{q}_{e}^{\prime}$ is a fixed point of the reversing symmetry (i.e., lies on the "mirror axis" $\theta=0$ ) implies that $\mathbf{q}_{e}^{\prime}$ is a reversible Lyapunov center [14,23], which is a marginally stable equilibrium point enclosed by a oneparameter family of periodic orbits.

As an example, Fig. 2(b) shows the phase portrait in $(y, \theta)$ plane for a swimmer with $d=10$ under constant input $\mathbf{u}_{e}$ $=(1,-1.0068)$, resulting in a marginally stable equilibrium point $\mathbf{q}_{e}^{\prime}=\left(9,0^{\circ}\right)$ with eigenvalues $\pm 0.77 i \times 10^{-3}$. The solid curves are trajectories of $(y, \theta)$ for various initial conditions [24]. The dashed curves correspond to configurations at which one of the spheres touches the wall, thus they bound the physically permissible region in $(y, \theta)$ plane. Small initial perturbations about $\mathbf{q}_{e}^{\prime}$ result in periodic orbits, corresponding to wave-like motion along the wall [Fig. 2(b)]. Larger perturbations result in trajectories under which $\theta$ grows mo- notonously, corresponding to spiral motion [Fig. 2(c)]. The additional equilibrium points, marked by $\times$ in Fig. 2(b), are saddle points corresponding to unstable motion parallel to the wall in a nearly-horizontal orientation $\left(\theta= \pm 79.85^{\circ}\right)$ [13].

Note that existence of periodic motion for rigid bodies in viscous fluid was already studied in classical works such as [25], as well as in the recent work [26] for larvae swimming in shear flow. In [27], the relation of periodic motion with reversing symmetry is formally established for particles sedimenting in a viscous fluid. A key contribution of our work is showing that the presence of a plane wall also induces a reversing symmetry, which, in turn, implies existence of periodic motions for a swimmer in quiescent fluid.

\section{2+1-SPHERE SWIMMER}

Since the reversing symmetry precludes the possibility of asymptotically stable motion parallel to the wall, our next goal is to break this symmetry by changing the structure of the swimmer. This is done by adding an unactuated sphere which is rigidly attached to the swimmer's body and forms an isosceles triangle of height $l$ with the two actuated spheres [Fig. 1(b)]. For this swimmer, steady motion parallel to the wall occurs at an orientation angle slightly shifted from $\theta=0$. As an example [13], for $d=10, l=2$, and $h=3$, a relative equilibrium point exists at $\mathbf{q}_{e}^{\prime}=\left(8.9866,-4.19^{\circ}\right)$ under input $\mathbf{u}_{e}=(-1,1.0086)$. The eigenvalues of the associated linearization matrix $\mathbf{A}$ are $\lambda_{1,2}=(-0.3984 \pm 1.5341 i) \times 10^{-3}$, hence $\mathbf{q}_{e}^{\prime}$ is asymptotically stable. Figures 3(a) and 3(b) show simulation results of $y(t)$ and $\theta(t)$ for this swimmer with constant input $\mathbf{u}=\mathbf{u}_{e}$ under initial perturbation of $\mathbf{q}^{\prime}(0)=\mathbf{q}_{e}^{\prime}$ $+\left(10,20^{\circ}\right)$. Figure $3(\mathrm{c})$ shows snapshots of the swimmer's motion along the wall. It is clearly seen that the swimmer is passively stabilized at $\mathbf{q}_{e}^{\prime}$ without applying any feedback.

An important observation is that this stability result is not necessarily limited to the specific swimmer model in Fig. 1(b). It may also hold for any swimmer with a constant shape that breaks its fore-aft symmetry properly. Therefore, our analysis can be viewed as an extension of the result in [19], discussing the hydrodynamic attraction of swimming microorganisms to walls, where the swimmer is now modeled as a constant-shape swimmer with broken fore-aft symmetry. Note that such a swimmer has a preferred direction of motion parallel to the wall, which is asymptotically stable [for example, swimming to the right in Fig. 1(b)]. Obviously, motion in the reversed direction is unstable and leads to migration of the swimmer away from the wall. This fact is somewhat analogous to the distinction between "pushers" and "pullers" in [19] according to the polarity of the force dipole.

\section{THREE-SPHERE SWIMMER}

As a final step, consider a swimmer with three actuated spheres. This swimmer possesses steady parallel motion at any desired orientation and wall separation, namely, the input $\mathbf{u}_{e}$ associated with a relative equilibrium at $\mathbf{q}_{e}^{\prime}=\left(y_{e}, \theta_{e}\right)$ is obtained by inverting $\mathbf{G}\left(\mathbf{q}^{\prime}\right)$ as

$$
\mathbf{u}_{e}\left(\mathbf{q}_{e}^{\prime}\right)=\mathbf{G}\left(\mathbf{q}_{e}^{\prime}\right)^{-1}\left(\begin{array}{lll}
1 & 0 & 0
\end{array}\right)^{T} .
$$




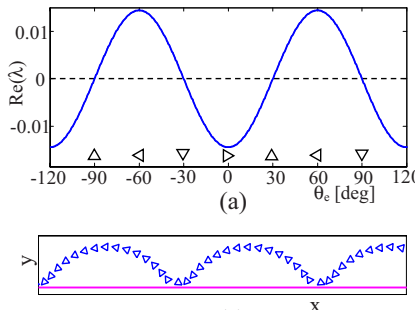

(c)

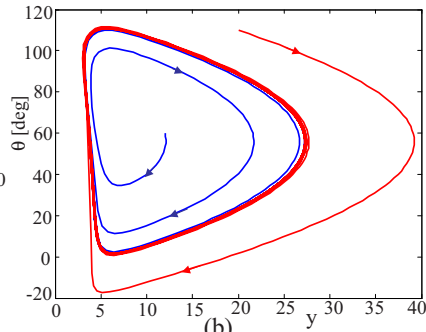

FIG. 4. (Color online) The three-sphere swimmer. (a) Real part of eigenvalues as a function of $\theta_{e}$. (b) Phase plot for $\theta_{e}=60^{\circ}$. (c) Snapshots of the periodic motion along the wall.

In the example of Fig. 1(c), the spheres are arranged in an equilateral triangle with edge length $d=5$. Fixing $y_{e}=7$ and expressing $\mathbf{u}_{e}$ as a function of $\theta_{e}$ according to Eq. (7), we numerically compute the eigenvalues of the associated linearization matrix $\mathbf{A}$, and their real part as a function of $\theta_{e}$ is shown in Fig. 4(a). The results, which are $120^{\circ}$-periodic due to the symmetry of the triangle, show regions of orientations $\theta_{e}$ for which parallel swimming is stable. Not surprisingly, the transitions from stability to instability occur at orientations $\theta_{e}= \pm 30^{\circ}$, at which the swimmer possesses fore-aft symmetry. Moreover, when $\theta_{e}$ is regarded as a parameter of the dynamical system $\dot{\mathbf{q}}^{\prime}=\mathbf{G}^{\prime}\left(\mathbf{q}^{\prime}\right) \mathbf{u}\left(\theta_{e}\right)$, the values $\theta_{e}$ $= \pm 30^{\circ}$, associated with a pair of imaginary eigenvalues, correspond to a Hopf bifurcation, indicating existence of periodic solutions [15]. Indeed, numerical simulation with $\theta_{e}$ $=60^{\circ}$, corresponding to an unstable equilibrium $\mathbf{q}_{e}^{\prime}$, reveals the existence of a stable periodic orbit, shown in $(y, \theta)$ plane in Fig. 4(b). Snapshots of the swimmer's periodic motion along the wall are shown in Fig. 4(c). Simulations for other values of $\theta_{e}$ indicate that whenever the steady parallel swimming is unstable, a stable periodic motion exists. Moreover, the periodic orbit encloses the region of attraction of the stable equilibrium $\mathbf{q}_{e}^{\prime}$ in the reversed motion (see [13] for details).

\section{CONCLUSION}

We have studied the dynamics and stability of low-Re swimmers with constant shape near a wall. We have shown that the reversing symmetry induced by the presence of the wall implies marginal stability and periodic motions. We then demonstrated how breaking the reversing symmetry can lead to passive stability of swimming parallel to the wall and showed the existence of stable periodic motion along the wall. Currently, we are building an experimental system in order to verify the theoretical results on a macroscale swimmer prototype in highly viscous silicon oil. An interesting open problem is the extension of the results to analyze the motion of shape-changing swimmers such as Purcell's threelink swimmer [9] near a wall in order to better model the true behavior of swimming microorganisms near surfaces.

\section{ACKNOWLEDGMENTS}

Y.O. was supported by the Fulbright Foundation and the Bikura Scholarship of the ISF. We thank the anonymous referees for their useful comments.
[1] T. J. Pedley and J. O. Kessler, Annu. Rev. Fluid Mech. 24, 313 (1992).

[2] G. Kosa et al., IEEE Trans. Rob. Autom. 23, 137 (2007).

[3] J. Happel and H. Brenner, Low Reynolds Number Hydrodynamics (Prentice-Hall, New Jersey, 1965).

[4] S. Kim and S. J. Karrila, Microhydrodynamics (ButterworthHeinemann, Boston, 1991).

[5] E. M. Purcell, Am. J. Phys. 45, 3 (1977).

[6] A. Shapere and F. Wilczek, J. Fluid Mech. 198, 557 (1989); J. E. Avron and O. Raz, New J. Phys. 10, 063016 (2008); J. Koiller, R. Montgomery, and K. Ehlers, J. Nonlinear Sci. 6, 507 (1996); S. D. Kelly and R. M. Murray, J. Rob. Syst. 12, 417 (1995).

[7] D. F. Katz, J. Fluid Mech. 64, 33 (1974).

[8] M. Ramia et al., Biophys. J. 65, 755 (1993).

[9] L. E. Becker et al., J. Fluid Mech. 490, 15 (2003); A. Najafi and R. Golestanian, Phys. Rev. E 69, 062901 (2004).

[10] A. M. Leshansky and O. Kenneth, Phys. Fluids 20, 063104 (2008).

[11] A. M. Leshansky et al., New J. Phys. 9, 145 (2007).

[12] A. Bernheim-Groswasser et al., Nature (London) 417, 308 (2002).

[13] See EPAPS Document No. E-PLEEE8-79-R08904 for a supplementary technical report. For more information on EPAPS, see http://www.aip.org/pubservs/epaps.html.

[14] J. S. W. Lamb and J. A. G. Roberts, Physica D 112, 1 (1998).
[15] J. Guckenheimer and P. Holmes, Nonlinear Oscillations, Dynamical Systems, and Bifurcations of Vector Fields (SpringerVerlag, New York, 1983).

[16] P. Frymier et al., Proc. Natl. Acad. Sci. U.S.A. 92, 6195 (1995); W. R. DiLuzio et al., Nature (London) 435, 1271 (2005).

[17] J. Cosson, P. Huitorel, and C. Gagnon, Cell Motil. Cytoskeleton 54, 56 (2003); B. M. Friedrich and F. Julicher, Proc. Natl. Acad. Sci. U.S.A. 104, 13256 (2007).

[18] L. J. Fauci and A. McDonald, Bull. Math. Biol. 57, 679 (1995).

[19] A. P. Berke, L. Turner, H. C. Berg, and E. Lauga, Phys. Rev. Lett. 101, 038102 (2008).

[20] Assuming that the hydrodynamic resistance of the thin body structure is negligible compared to that of the spheres.

[21] J. W. Swan and J. F. Brady, Phys. Fluids 19, 113306 (2007).

[22] J. E. Marsden, Lectures on Mechanics (Cambridge University Press, New York, 1992).

[23] R. L. Devaney, Trans. Am. Math. Soc. 218, 89 (1976).

[24] Note that the phase portrait is $360^{\circ}$ periodic in $\theta$ and has a reflection symmetry about the lines $\theta=0^{\circ}$ and $\theta=180^{\circ}$, as implied by the reversing symmetries of the system.

[25] G. B. Jeffery, Proc. R. Soc. London, Ser. A 102, 161 (1922); R. E. Caflisch et al., Phys. Fluids 31, 3175 (1988).

[26] G. Zilman et al., Mar. Biol. (Berlin) 154, 1 (2008).

[27] M. Golubitsky et al., SIAM J. Appl. Math. 51, 49 (1991). 